\title{
Ligation of the external iliac artery for post-transplant nephrectomy bleeding
}

\author{
M. W. BROWN \\ M.B., Ch.B.
}

J. A. BRadley

F.R.C.S.

\author{
D. N. H. HAMilton \\ F.R.C.S., Ph.D.
}

University Department of Surgery, Western Infirmary, Glasgow G11 6NT

\begin{abstract}
Summary
Two patients who had transplant nephrectomy performed for rejection of a previously transplanted human cadaver allograft kidney subsequently developed complications due to a false aneurysm of the external iliac artery. Treatment was successfully carried out by ligation of the external iliac artery without any significant ischaemic problems in the corresponding lower limb.
\end{abstract}

\section{Introduction}

Human cadaver kidneys are usually transplanted to the iliac fossa. The donor renal artery is anastomosed either end-to-end to the recipient internal iliac artery or, especially if there is more than one renal artery, an aortic patch from the donor vessel is sutured end-to-side to the recipient external iliac artery. If this patch method is used and the kidney rejects, the kidney is removed by ligating and dividing the donor artery and vein leaving the donor patch behind. Rarely, the residual patch may be weakened, by infection at the suture line or by rejection. Two cases of major bleeding from such a cause are reported here.

\section{Case 1}

A 24-year-old male patient on regular dialysis treatment for chronic renal failure due to chronic glomerulonephritis received a cadaver kidney allograft in July 1980, which was placed in the left iliac fossa. The donor renal artery's aortic patch was anastomosed end-to-side to the recipient's external iliac artery. One week later, there were signs of early acute rejection and one month later graft function failed. The patient had also developed a discharging transplant wound infection. Transplant nephrectomy was therefore carried out with ligation of the donor artery and vein, leaving short lengths of donor vessel in place. Histology confirmed an acute vascular rejection of the kidney.
During the next two weeks, the infected transplant wound continued to ooze blood and discharge moderate amounts of pus, which grew a coagulase negative Staphylococcus albus sensitive to flucloxacillin. Antibiotic treatment was started, but two transient 0 episodes of brisk haemorrhage from the wound occurred at this time. Several days later a further major wound haemorrhage occurred and a leakage from the external iliac artery as a result of weakening of the donor patch was diagnosed.

Emergency surgery was undertaken and contro of the haemorrhage was achieved by two balloo embolectomy catheters inserted retrogradely fror the femoral artery in the groin, with the balloons positioned in the external iliac above and below the likely site of the leak. Since a direct approach to the leak was judged hazardous it was decided simply to ligate the leaking segment of iliac artery.

Through an abdominal incision, the external iliac artery was ligated below the origin of the internal iliac. Then the femoral artery was displayed at the groin and after checking that satisfactory backflow up the femoral artery existed, it was ligated above the origin of the profunda artery. Perfusion of the leg was unaffected though no peripheral pulses could be detected. The patient subsequently made an uneventful recovery, returned to regular dialysis treatment and by 6 months the peripheral pulses had returned.

\section{Case 2}

A 54-year-old male on regular haemodialysis treatment for chronic renal failure due to chronic $N$ glomerulonephritis, received a cadaver kidney graft $\mathrm{c}$ placed in the left iliac fossa in January 1981. The donor artery had an aortic patch which was anastomosed end-to-side to the recipient's external iliac artery.

Within 10 days there was evidence of rejection and biopsy confirmed an acute rejection. Twenty-one 
days post-transplant the graft was removed, the vascular pedicle being ligated, leaving behind short lengths of donor vessels.

Over the next week, the patient developed an infected nephrectomy wound and culture revealed a heavy growth of Staphylococcus aureus sensitive to cloxacillin, which was therefore commenced. One week later a palpable mass developed beneath the wound with marked local tenderness. A major haemorrhage then occurred from the wound. A leak from the site of the donor patch of the external iliac artery was diagnosed and the patient resuscitated. To avoid the dangers of a direct approach to the bleeding area it was decided to use the same technique as in Case 1, namely tying off the segment of artery bearing the patch. Laparotomy was carried out and this revealed blood tracking up the retroperitoneal tissue from the large haematoma in the transplant site. The external iliac artery was ligated at its origin and, through a groin incision, the common femoral artery was ligated above the origin of the profunda artery. Perfusion in the left leg was initially slightly diminished, but was normal the next day. The patient made an uneventful recovery apart from a minor wound infection and was recommenced on regular haemodialysis. Examination 6 months later showed that the peripheral pulses had returned.

\section{Discussion}

Major arterial bleeding from the remaining arterial patch following transplant nephrectomy is an uncommon event; we have only encountered two cases in over 100 transplant nephrectomies. In both these cases the haemorrhage was successfully controlled by ligation of the external iliac artery, below the origin of the internal iliac artery, plus ligation of the femoral artery, proximal to the origin of the profunda femoris, to control back flow into the segment containing the patch. By ligating the vessel in this manner, retrograde flow from the profunda femoris via collaterals can enter the superficial femoral artery allowing satisfactory distal perfusion. In order to minimize the risks of distal ischaemia care should be taken to ensure that the sites of ligation do not interfere with blood flow in the internal iliac and profunda femoris arteries.

Although it was not possible to define the bleeding point precisely in either case, it seems probable that the bleeding occurred from the donor renal artery patch, either because the patch rejected or more likely because it was weakened by infection. If infection is the cause, this probably occurs following transplant nephrectomy and if this is carried out in the presence of a contaminated wound, antibiotic prophylaxis based on culture of any discharge may help to prevent this potentially fatal complication. Angiography was not performed in the present cases since it would have produced unnecessary delay, but a previous report has shown that ruptured mycotic aneurysms of the iliac vessels may occur following transplant nephrectomy (Kyriakides, Simmons and Najarian, 1976).

Treatment by ligation of the external iliac artery seemed best since infection in the wound would have made repair of the defect in the external iliac artery risky and possibly liable to leak again later. This method of treatment is not new, but we believe that it is not a widely appreciated therapeutic option. The largest previous report (Owens et al., 1979) describes 9 cases where ligation of a major pelvic vessel (in 7 cases this was the external iliac artery) was performed in patients who developed a major haemorrhage from a transplant site. In 4 cases this followed attempts at direct repair of the defect. These initial attempts to directly explore and repair the bleeding vessels in these patients were usually unsuccessful and ligation of the major vessels as the first operation was a successful treatment and was not usually associated with significant limb ischaemia. Measurements of arterial blood flow were not made in our two cases, but both showed a return of peripheral pulses by 6 months and neither experienced definite claudication although the first patient occasionally complained of muscular discomfort in the posterior thigh when going upstairs. Thus in our limited experience and that of others, (Owens et al., 1979; M. Bewick, unpublished observations), if arterial bleeding occurs from the site of a transplant nephrectomy, ligation of the parent artery proximally and distally to the site of haemorrhage may be the best treatment. If signs of ischaemia persist we would recommend a delayed bypass procedure following eradication of any infection.

\section{Acknowledgments}

We would like to thank Mr R. O. Quin for his helpful comments, and Dr J.M. Boulton-Jones and Dr J. D. Briggs for permission to report these cases.

\section{References}

Kyriakides, G.K., Simmons, R.L. \& Najarian, J.S. (1976) Mycotic aneurysms in transplant patients. Archives of Surgery, 111, 472.

OWens, M.L., Wilson, S.E., Maxwell, J.G., Bordner, A., Smith, R. \& Ehrlich, R. (1979) Major arterial haemorrhage after renal transplantation. Transplantation, 27, 285. 